\title{
ERRATUM
}

\section{Human Smart Cities}

\section{Rethinking the Interplay between Design and Planning}

\author{
Grazia Concilio and Francesca Rizzo \\ (C) Springer International Publishing Switzerland 2016 \\ G. Concilio, F. Rizzo (eds.), Human Smart Cities, Urban and Landscape Perspectives, \\ DOI 10.1007/978-3-319-33024-2
}

DOI 10.1007/978-3-319-33024-2_16

Volume number, volume 19 included in the PDF.

The updated original online version for this book can be found at DOI 10.1007/978-3-319-33024-2 